\title{
Screening of Pearl Millet (Pennisetum glaucum) Genotypes against Smut Caused by Moesziomyces penicillariae (Bref) Vanky
}

\author{
Annie Khanna*, Kushal Raj and Narender Singh
}

Department of Plant Pathology, College of Agriculture, haudhary Charan Singh Haryana Agricultural University, Hisar 125004, Haryana, India

*Corresponding author

\section{A B S T R A C T}

\section{Keywords}

Smut severity, Germplasm, Screening, Pearl millet

\section{Article Info}

\section{Accepted:}

20 April 2018

Available Online:

10 May 2018

\begin{abstract}
Smut caused by Moesziomyces penicillariae is one of the major pearl millet diseases in India. in order to find out cost effective, economical and ecofriendly method to manage the disease attempts were made to identify the resistant sources of smut by germplasm screening. The field screening involved the artificial inoculation of the germplasm. Observations on smut severity were recorded 15-20 days after inoculation using smut severity (\%) rating scale. Amongst 239 pearl millet genotypes comprising of initial varietal trial (IVT), advanced varietal trial (AVT) and final yield trial (FYT) were screened for their reaction to smut and identification of the resistant source against pearl millet smut. It was observed that 52 genotypes were completely free from smut, 93 genotypes showed less than 10 per cent smut severity, 66 genotypes showed $10-25$ per cent smut severity and 27 genotypes showed more than 25 per cent smut severity.
\end{abstract}

\section{Introduction}

Pearl millet [Pennisetum glaucum (L.) R. Br.], also known as bajra, bajri etc is the important cereal crop for the population living in drought prone arid and semi arid regions of less developed countries in the world. Recent archaeobotanical research has confirmed the presence of domesticated pearl millet on the Sahel zone of northern Mali between 25002000 BC (Manning et al., 2010). In India this crop is grown in the states of Haryana, Rajasthan, Madhya Pradesh, Andhra Pradesh, Tamil Nadu, Gujarat and Karnataka. Pearl millet is the major kharif crop of Haryana with area of 5.10 lakh ha and productivity is 1850 $\mathrm{kg} / \mathrm{ha}$. Though the pearl millet yield is increased with the introduction of hybrids but it has become susceptible to many diseases. Amongst those smut is one of the major diseases in areas where pearl millet is cultivated. Moesziomyces penicillariae (Tolyposporium penicillariae), causes smut disease and has been observed in the pearl millet growing regions. Smut in pearl millet reduces the grain yield. Use of host plant resistance is one of the best measures for the integrated disease management. The present investigation was therefore undertaken to evaluate the pearl millet genotypes to find out resistance against smut.

Materials and Methods 
During kharif, 2016 the experiment for screening was conducted with a total of 239 pearl millet genotypes with two replications in randomized bock design (RBD), with row length of 5 meter. The test entries were sown on 16-07-2016 with row to row spacing of 50 $\mathrm{cm}$ and plant to plant distance of $10 \mathrm{~cm}$ in each row. For artificial screening fifteen earhead at boot leaf stage of each entry were randomly selected from each replication and were inoculated by aqueous sporidial suspension of Moesziomyces penicillariae. The sporidial suspension $\left(10^{6}\right.$ sporidia/ml) was prepared by dipping the smut infected earheads in water for 24 hours so that spores germinate before inoculation. After inoculation of the earheads at boot leaf stage, the earheads were covered with a parchment paper bag and water was sprinkled regularly to maintain high humidity. The parchment paper bags were removed 15-20 days after inoculation and smut severity was recorded using smut severity (\%) rating scale as given by Thakur and King (1988) (Fig. 1).

\section{Results and Discussion}

In order to manage the disease, incorporation of resistance in suitable genotype is the demand of current era as it is considered as the most economical method. In the present study, amongst 239 genotypes screened against smut of pearl millet, 52 genotypes were free from smut, 94 genotypes exhibited smut severity $<10 \%, 66$ genotypes showed smut severity 1025 per cent and in remaining 27 genotypes smut severity was more than $25 \%$. Thakur et al., (1986) screened 1500 pearl millet accessions against $M$. bullatus for 1-6 years of multi location testing in India and West Africa and found that six germplasm accessions viz., SSC FS 252-S-4, ICI 7517-S-1, ExB 132-2-S5-2-DM-1, ExB 46-1-2-S-2, ExB 112-1-S-1-1 and P-489-S-3 and four newly developed, resistant, agronomically elite lines viz., ICMPS 100-5-1, 900-9-3, 1600-24 and 20005-2 showed consistently high levels of resistance (Table 1-3).

Fig.1 Smut severity (\%) rating scale as given by Thakur and King (1988)

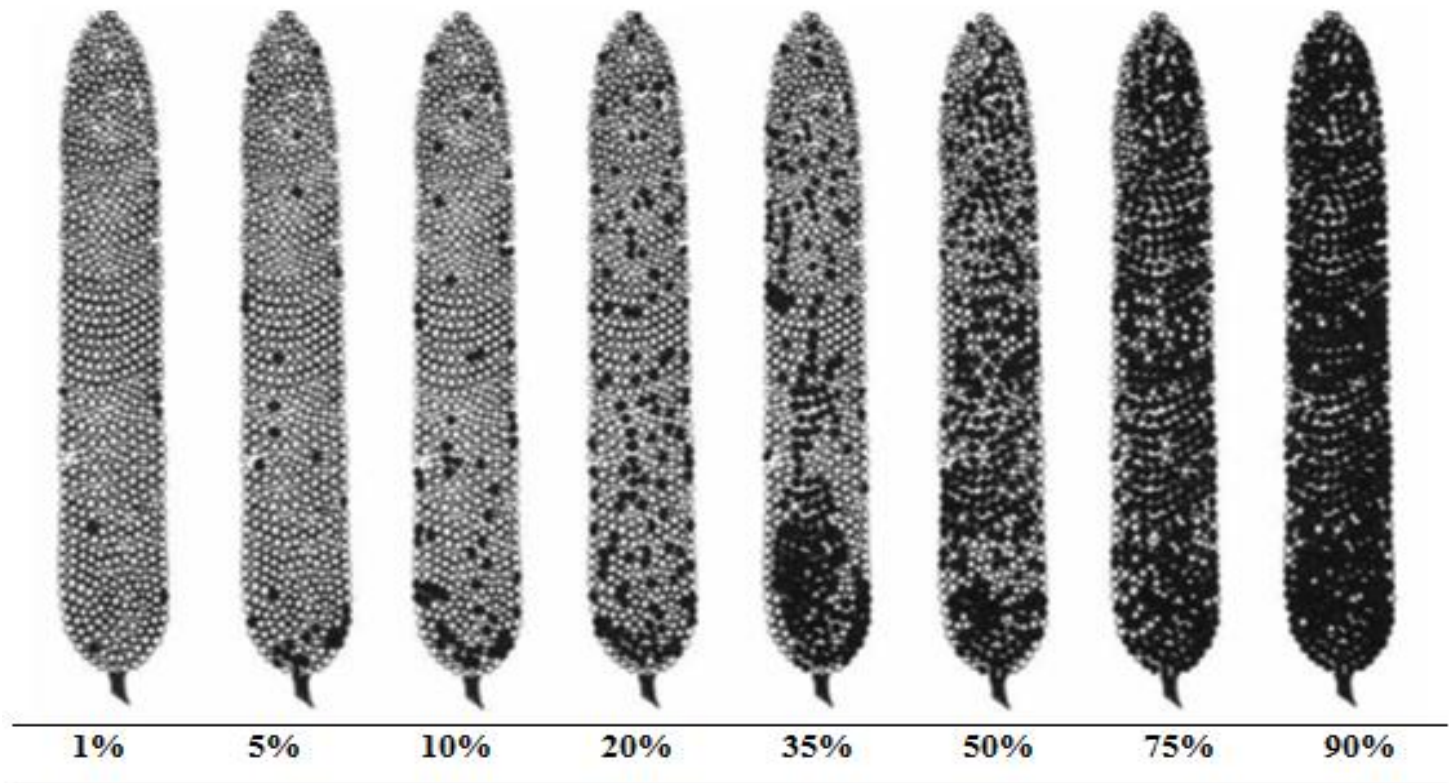


Table.1 Screening of pearl millet initial varietal trial (IVT) genotypes against smut caused by Moesziomyces penicillariae

\begin{tabular}{|c|c|c|}
\hline $\begin{array}{l}\text { Smut } \\
\text { severity \% }\end{array}$ & Genotype & $\begin{array}{l}\text { Total number of } \\
\text { genotypes }\end{array}$ \\
\hline FREE & $\begin{array}{l}\text { MH2187, MH2188, MH2189, MH2202, MH2215, MH2228, } \\
\text { MH2231, MH2241, MH2242, MH2249, MH2256, MH2260, } \\
\text { MH2263, MH2266, MH2268, MH2272, MH2273, MH2281, } \\
\text { MH2285, MH2287, RHB177, KBH108, NBH5061, Kaveri Super } \\
\text { Boss, RAJ171, ICMV } 221\end{array}$ & 26 \\
\hline $0-10$ & $\begin{array}{l}\text { MH2190, MH2203, MH2211, MH2214, MH2216, MH2219, } \\
\text { MH2223, MH2227, MH2233, MH2236, MH3337, MH2238, } \\
\text { MH2239, MH2240, MH2245, MH2246, MH2247, MH2248, } \\
\text { MH2250, MH2251, MH2252, MH2253, MH2254, MH2255, } \\
\text { MH2257, MH2258, MH2259, MH2261, MH2264, MH2265, } \\
\text { MH2267, MH2271, MH2276, MH2278, MH2279, MH2282, } \\
\text { MH2283, MH2284, MH2288, MP574,MP575, MP577, MP579, } \\
\text { MP580, MP581, 86M01, GH905, MPMH17,Pratap, NBH5767, } \\
\text { PAC909, 86M86 }\end{array}$ & 52 \\
\hline $10-25$ & $\begin{array}{l}\text { MH2191, MH2192, MH2194, MH2195, MH2199, MH2201, } \\
\text { MH2205, MH2208, MH2209,MH2212, MH2213, MH2217, } \\
\text { MH2218, MH2220, MH2221, MH2222, MH2224, MH2226, } \\
\text { MH2229, MH2230, MH2232, MH2234, MH2235, MH2243, } \\
\text { MH2244, MH2262, MH2269, MH2274, MH2275, MH2277, } \\
\text { MH2280, MH2286, MP576, MP578, MP-7792, JBV2, ICMV155 }\end{array}$ & 37 \\
\hline$>25$ & $\begin{array}{l}\text { MH2193, MH2196, MH2197, MH2198, MH2200, MH2204, } \\
\text { MH2206, MH2207, MH2210, MH2225, MH2270, Dhanshakti, } \\
\text { ICMH356, HHB67 Improved }\end{array}$ & 14 \\
\hline
\end{tabular}

Table.2 Screening of pearl millet advanced varietal trial (AVT) genotypes against smut caused by Moesziomyces penicillariae

\begin{tabular}{|c|l|c|}
\hline $\begin{array}{l}\text { Smut } \\
\text { severity \% }\end{array}$ & Genotype & $\begin{array}{c}\text { Total number of } \\
\text { genotypes }\end{array}$ \\
\hline \multicolumn{1}{|c|}{ FREE } & $\begin{array}{l}\text { MH2035, MH2047, MH2078, MH2106, MH2129, MH2137, } \\
\text { MH2151, MH2162, MH2172, MH2174, MP562, PRATAP, } \\
\text { NBH5061, Kaveri Super Boss }\end{array}$ & \\
\hline $0-10$ & $\begin{array}{l}\text { MH1993, MH1996, MH2008, MH2010, MH2039, MH2053, } \\
\text { MH2076, MH2087, MH2098, MH2099, MH2101, MH2107, }\end{array}$ & \\
& $\begin{array}{c}\text { MH2113, MH2130, MH2132, MH2149, MH2154, MH2155, } \\
\text { MH2173, RHB177, HHB67 Improved, MPMH17, RHB173, }\end{array}$ & \\
& NBH5767, PAC909, 86M86, 86M64, Pusa Composite 383, & \\
\hline ICMV221, ICMV155 & \\
\hline 10-25 & MH1998, MH2024, MH2072, MH2077, MH2082, MH2088, \\
& MH2097, MH2110, MH2114, MH2123, MH2134, MH2141, & \\
& MH2147, MH2156, MH2180, MP570, GHB905, GHB744, \\
& KBH108, MP-7792, RAJ171, Dhanshakti & \\
\hline$>25$ & MH2089, MH2118, MH2178, MH2179, MH2183, MH2185, & \\
& MP 552, MP 571, ICMH 356, GHB 558 & \\
\hline
\end{tabular}


Table.3 Screening of pearl millet final yield trial (FYT) genotypes against smut caused by Moesziomyces penicillariae.

\begin{tabular}{|c|l|c|}
\hline $\begin{array}{l}\text { Smut } \\
\text { severity \% }\end{array}$ & Genotype & $\begin{array}{l}\text { Total number of } \\
\text { genotypes }\end{array}$ \\
\hline FREE & $\begin{array}{l}\text { HHB234, HHB 67 Improved, GHB905, MPMH17, RHB173, } \\
\text { GHB558, MP-7792, Nandi 61, 86M88, Dhanshakti, Raj 171, } \\
\text { JBV 2 }\end{array}$ & 12 \\
\hline $0-10$ & $\begin{array}{l}\text { HHB226, HHB197, HHB223, KBH108, GHB732, Kaveri } \\
\text { Suoer Boss, NBH 5767, Pratap(MH 1642), NBH 5061, MBC } \\
\text { 2, ICMV 155 }\end{array}$ & \\
\hline $10-25$ & $\begin{array}{l}\text { GHB 538, 86M01, 86M86, Proagro 9444, PAC 909, Pusa } \\
\text { Composite 383, ICMV 221 }\end{array}$ & \\
\hline$>25$ & RHB 177, GHB 744, 86M64 & 7 \\
\hline
\end{tabular}

Pandya and Bartaria (2000) evaluated several pearl millet lines under artificial inoculation and identified ICMB 92888, ICMB 92777 and IP 19874 as a source of smut resistance. Choursia (2007) evaluated 138 entries against smut and observed that only one entry $\mathrm{MH}$ 1317 remained absolutely free from smut while nineteen entries were in the category of 5.1- 10 per cent smut severity and maximum severity was recorded in $\mathrm{MH}$ 1391. Based on screening of pearl millet entries against smut, the new hybrids are promoted for cultivation from time to time.

In conclusion, screening of pearl millet genotypes was done during kharif 2016, in IVT genotypes found free from smut were MH2187, MH2188, MH2189, MH2202, MH2215, MH2228, MH2231, MH2241, MH2242, MH2249, MH2256, MH2260, MH2263, MH2266, MH2268, MH2272, MH2273, MH2281, MH2285, MH2287, RHB177, KBH108, NBH5061, Kaveri Super Boss, RAJ171 and ICMV 221 whereas in AVT fourteen genotypes namely MH2035, MH2047, MH2078, MH2106, MH2129, MH2137, MH2151, MH2162, MH2172, MH2174, MP562, PRATAP, NBH5061, Kaveri Super Boss were free from smut. Amongst thirty three genotypes screened against smut in FYT eleven genotypes viz.,
HHB234, HHB 67 Improved, GHB905, MPMH17, RHB173, GHB558, MP-7792, Nandi 61, 86M88, Dhanshakti, Raj 171, JBV 2 were found resistant to smut. Thus, the resistant sources identified can be used for future breeding programme.

\section{References}

Choursia, A. (2007). Studies on smut of pearl millet with special reference to its chemical management M.Sc. Thesis submitted to the JNKVV, Jabalpur (M.P.), Campus, College of Agriculture, Gwalior. pp. 18-22.

Manning, K., Ruth, P., Higham, T. Schwenniger, J. L. and Fuller, D. Q. (2010). 4500 year old domesticated pearl millet (Pennisetum glaucum) from the Tilemsi valley, Mali: new insights into an alternative cereal domestication pathway. Journal of Archaeological Science, 38 (2): 312-322.

Pandya, R.K. and Bartaria, A.M. (2000). Evaluation of pearl millet lines for smut in Northern Madhya Pradesh. Journal of Mycology and Plant Pathology, 30: 278.

Rai, K.N., Thakur, R.P. and Rao, A.S. (1998). Registration of smut resistant pearl millet parental lines ICMA 88006 and 
ICMB 88006. Crop Science, 38: 575- Thakur, R.P., Rao, K.V., Williams, R.J., 576.

Thakur, R.P. and King, S.B. Gupta, S.C.D.P., Nafade, S.D. Soundarum, N.V., Forwd, J.A. and (1988).Registration of six smut resistance germplasm of pearl millet. Plant Pathology, 30: 557-563. Guthrie, E.J. (1986). Identification of stable resistance to smut in pearl millet. Plant Disease, 70: 38-41.

\section{How to cite this article:}

Annie Khanna, Kushal Raj and Narender Singh 2018. Screening of Pearl Millet (Pennisetum glaucum) Genotypes against Smut Caused by Moesziomyces penicillariae (Bref) Vanky. Int.J.Curr.Microbiol.App.Sci. 7(05): 2865-2869. doi: https://doi.org/10.20546/ijcmas.2018.705.333 